ORIGINAL ARTICLE

\title{
Quality of life in chronic illness: perceptions of parents and paediatricians
}

\author{
A J Janse, G Sinnema, C S P M Uiterwaal, J L L Kimpen, R J B J Gemke
}

Arch Dis Child 2005;90:486-491. doi: 10.1136/adc.2004.051722

See end of article for authors' affiliations

.....................

Correspondence to:

Dr G Sinnema

Department of Paediatric

Psychology, KA.00.004.0,

Wilhelmina Children's

Hospital, University

Medical Centre Utrecht,

PO Box 85090,3508 AB

Utrecht, Netherlands;

G.Sinnema@wkz.azu.n

Accepted

24 September 2004

\begin{abstract}
Aims: To investigate the differences in perception of quality of life between parents of chronically ill children and paediatricians at diagnosis and follow up. Quality of life was assessed using the (HUI3). Methods: Longitudinal study (July 1999-January 2002) of 37 paediatricians and 181 parents of patients (children aged 1-17 years) with cystic fibrosis admitted for a pneumonia or patients with newly diagnosed acute lymphatic leukaemia, juvenile idiopathic arthritis, or asthma. Main outcome measure was percentage agreement on the attributes of the HUI3 between parents and paediatricians.

Results: Differences in perception of health and wellbeing between paediatricians and parents of children with a chronic disease were found, not only at diagnosis but also after a period of follow up. Differences were particularly clear in the subjective attributes emotion (range of agreement 28-68\%) and pain/ discomfort (range of agreement 11-33\%). In all patient groups, at baseline and follow up, the paediatrician assessed the patient to have less pain/discomfort in comparison to the parents. Despite a prolonged patient- paediatrician relationship, differences at follow up did not decrease compared to baseline.

Conclusion: At the onset of a chronic disease, but also after a period of follow up, quality of life of paediatric patients may be misunderstood by healthcare professionals, especially in the subjective attributes. Systematic assessment of quality of life may contribute to better understanding between physicians and parents.
\end{abstract}

$\mathrm{T}$ monitor the effectiveness of clinical practice and to determine the efficacy of new treatment strategies general indicators of outcome are essential. Physicians predominantly use conventional clinical, laboratory, and radiological measures to assess the success of an intervention and implicitly estimate the wellbeing of the patient. In addition to these measures the importance of quality of life (QoL) assessment is increasingly recognised. In randomised clinical trials QoL is becoming an important secondary outcome. ${ }^{1-5}$ As improved treatment leads to substantial gains in survival rates (for example, in most cancers and cystic fibrosis), the prevalence of children with a chronic disease is increasing. ${ }^{6}$ Differences in perception of the wellbeing of patients between patients and their physicians may interfere with the effectiveness of treatment. ${ }^{7}$ Little is known in the literature about the differences in perception of wellbeing between paediatric patients and physicians. A recent metaanalysis showed that on more objective attributes (sensation, self-care, and mobility) the agreement between parents and physicians was higher than on attributes with a subjective nature (emotion, cognition, and pain/discomfort). ${ }^{8}$ However, none of the reviewed studies revealed the direction of the differences in perception of QoL between parents and physicians.

The aim of the present longitudinal study was to analyse the differences in perception of QoL between parents of chronically ill children and paediatricians at diagnosis and follow up, and to describe the direction of these differences. QoL was assessed using the Health Utilities Index mark 3 (HUI3). In children under the age of 10 years, parents generally act as a proxy for their child. Children 10 years and older are capable of giving reliable answers about their health status. However, until the age of 17 years, parents still have a crucial role in medical decisions concerning their child. In these decisions, parents rely on their perception of the health status of their child. Therefore, parents were asked to act as a proxy and to assess the QoL of their children. Patients with acute lymphoblastic leukaemia (ALL) were chosen because the HUI has been successfully used in this group..$^{9-11}$ Patients with juvenile chronic arthritis (JIA), cystic fibrosis (CF), and asthma were included because our hospital aims to be a centre of excellence for these diseases. Furthermore, we expected the attributes of the HUI3 to match well with major complaints of these patients.

We hypothesised that differences in QoL outcomes between parents and paediatricians would be greatest for the subjective attributes. We had no a priori expectations about paediatricians over- or underestimating QoL in comparison with parents of chronically ill children.

\section{METHODS}

\section{Patients}

Patients were enrolled at four tertiary care centres (Wilhelmina Children's Hospital, University Medical Centre Utrecht, Utrecht; Sophia Children's Hospital, Erasmus University Medical Centre, Rotterdam; Emma Children's Hospital, Academic Medical Centre, Amsterdam; and Asthma Centre Heideheuvel, Hilversum) in the Netherlands during the period July 1999 to January 2002. Eligibility criteria included children admitted with newly diagnosed ALL, children with CF admitted for pneumonia (CF-adm), children with the diagnosis of JIA or asthma and their first visit to the outpatient clinic, aged $1-17$ years (patients with asthma, 4-17 years old), and the ability of the parents to understand and read the Dutch language.

Abbreviations: ALL, acute lymphoblastic leukaemia; CF, cystic fibrosis; CF-adm, children with CF admitted for pneumonia; HUI3, Health Utilities Index mark 3; JIA, juvenile chronic arthritis; QoL, quality of life 


\section{Data collection}

QoL was assessed using a 42 item interview questionnaire (baseline) and a 15 item self-complete questionnaire (follow up) developed for parents. At baseline parents completed the interview questionnaire administered by the principal investigator (AJJ). The interview was completed during the first week following admission (ALL, CF-adm), or after the first visit to the paediatricians in the outpatient clinic (JIA, asthma). The follow up assessment was completed after induction therapy, before the start of methotrexate according to the SNWLK-ALL-9 protocol (ALL), ${ }^{12}$ 6-8 weeks after admission (CF-adm), three months after the first visit (asthma) or six months after the first visit (JIA) in the outpatient clinic. The follow up interval for each disease was different and determined by the time needed for stabilisation of the initial therapeutic effect according to consensus reached during meetings with clinical experts. In patients with ALL the follow up time was determined by the SNWLK protocol. ${ }^{12}$ Following the consultation or admission of the patient, the paediatricians completed the Multiattribute Health Status classification System HUI3 (described by Feeny et al). ${ }^{13}$ The paediatricians completed the HUI3 independently from the parents. Parents and paediatricians were asked to consider the health status of each patient for the preceding period of four weeks.

Information from these questionnaires was converted to health state vectors in the HUI3 formats by an established algorithm. ${ }^{14}$ The Health Utilities Index mark 2 and mark 3 (HUI2 and HUI3) are generic multiattribute health status classification systems. ${ }^{13}$ They have been used in a number of clinical studies of children with cancer, ${ }^{15-25}$ extremely low birth weight infants, ${ }^{26}{ }^{27}$ and survivors of paediatric intensive care. ${ }^{28}$ The HUI3 has been described in detail by Feeny and colleagues. ${ }^{13}$ Briefly, it consists of eight attributes. Each attribute consists of 5-6 levels representing the range of functioning from normal (1) to severely impaired (5 or 6). The attributes forming the HUI3 system are vision (1-6), hearing (1-6), speech (1-5), ambulation (1-6), dexterity (1-6), emotion (1-5), cognition (1-6), and pain/discomfort (1-5). For example, the attribute emotion represents the following levels: level 1, happy and interested in life; level 2, somewhat happy; level 3, somewhat unhappy; level 4, very unhappy; and level 5, so unhappy that life is not worthwhile.

\section{Consent and ethics approval}

Written informed consent was obtained from all parents and of patients over 12 years of age who agreed to participate in the study. The medical ethics committees of all participating centres approved the study protocol.

\section{Table 1 Patients' characteristics}

\begin{tabular}{ll}
\hline Characteristic & \\
\hline Pairs HUI all domains complete & $181 / 145$ \\
Mean age first assessment (SD) in years & $7.9(4.3)$ \\
Percentage male/female diagnosis (follow up) & $83(70) / 54$ (75) \\
Diagnosis (follow up); no. of complete pairs & \\
ALL & $47(40)$ \\
Asthma & $50(37)$ \\
CF & $39(30)$ \\
JIA & $45(38)$ \\
Mean duration follow up (weeks) & 7 \\
ALL & 14 \\
Asthma & 9 \\
CF & 28 \\
JIA &
\end{tabular}

ALL, acute lymphatic leukaemia; CF, cystic fibrosis admitted for pneumonia; JIA, juvenile idiopathic arthritis.

\section{Statistical analysis}

Patients were divided into four groups based on their diagnosis. For each group of patients the following procedure was used.

Absolute differences between physicians and parents for each attribute at baseline and at follow up were calculated using the formula: attribute level physician - attribute level parent. The outcome was dichotomised into presence (" 1 ") or absence (" 0 ") of difference between physician and parent. All outcomes zero become " 0 ", all negative and positive outcomes become " 1 ". Next, the percentage agreement between pairs of physicians and parents for each attribute were calculated exactly. Subsequently, the proportions of differences between parents and physicians ( $=$ percentage disagreement/100) at baseline $\left(P_{1}\right)$ and at follow up $\left(P_{2}\right)$ for each attribute were calculated. The differences in proportions at baseline and follow up were given by $P_{1}-P_{2}, 95 \%$ CI were calculated using the formula $P_{1}-P_{2} \pm 1.96^{*} S E\left[P_{1}-P_{2}\right] .{ }^{29} 95 \%$ CIs for differences in proportions that did not include 0 were considered statistically significant. A non-parametric test for paired samples (McNemar test) was used to test the null hypothesis that differences in perception between parents and physicians were the same at baseline and at follow up. Baseline differences in perception for pairs with and without missing data at follow up were tested using a $\chi^{2}$ test (for a two way frequency table). A p value $<0.05$ was considered statistically significant. The change in the differences (delta) for each attribute was calculated for all pairs by subtracting the absolute differences at follow up from the absolute differences at baseline.

A $10 \%$ random sample of all the collected data was taken (SPSS version 10.0), entered twice into the computer by two different persons, and verified (SPSS data entry builder version 1.0). The error rate was acceptable with $0.33 \%$. The SPSS statistical software (version 10.0) was used for all calculations.

\section{RESULTS}

\section{Study sample}

At baseline the parents of 185 of the 187 eligible children gave consent to participate. Reasons for refusal were the feeling of interference with the disease (JIA, $\mathrm{n}=1$ ) and psychological distress when faced with the diagnosis ALL $(n=1)$. Four other patients were excluded from further analysis because the paediatricians did not return the questionnaire. The final baseline analysis included 181 patients. Complete pairs at follow up were available for 145 of the 181 patients. Reasons for failure to participate at follow up were death ( 1 patient), no appointment or not showing up at the outpatient clinic (26 patients), and no returned questionnaires ( 6 patients and 3 physicians). Table 1 shows characteristics of the study group.

\section{Differences between parents and paediatricians at diagnosis and follow up}

For each group of patients the percentage of pairs with exact agreement between parents and physicians is given in fig 1 . For all patient groups the agreement for the attributes vision and hearing were above $90 \%$, in contrast to the agreement for the attributes pain/discomfort and emotion, which were low in all groups. There were no baseline differences for patients without and with missing data. The proportion of differences for the attribute pain/discomfort in patients with asthma and the attributes ambulation and emotion in patients with JIA were significantly lower at follow up than at baseline (table 2). For all other attributes no significant differences were found in the proportion of differences at baseline versus at follow up. Figure 2 shows the direction of the differences in QoL perception between paediatricians and parents. 

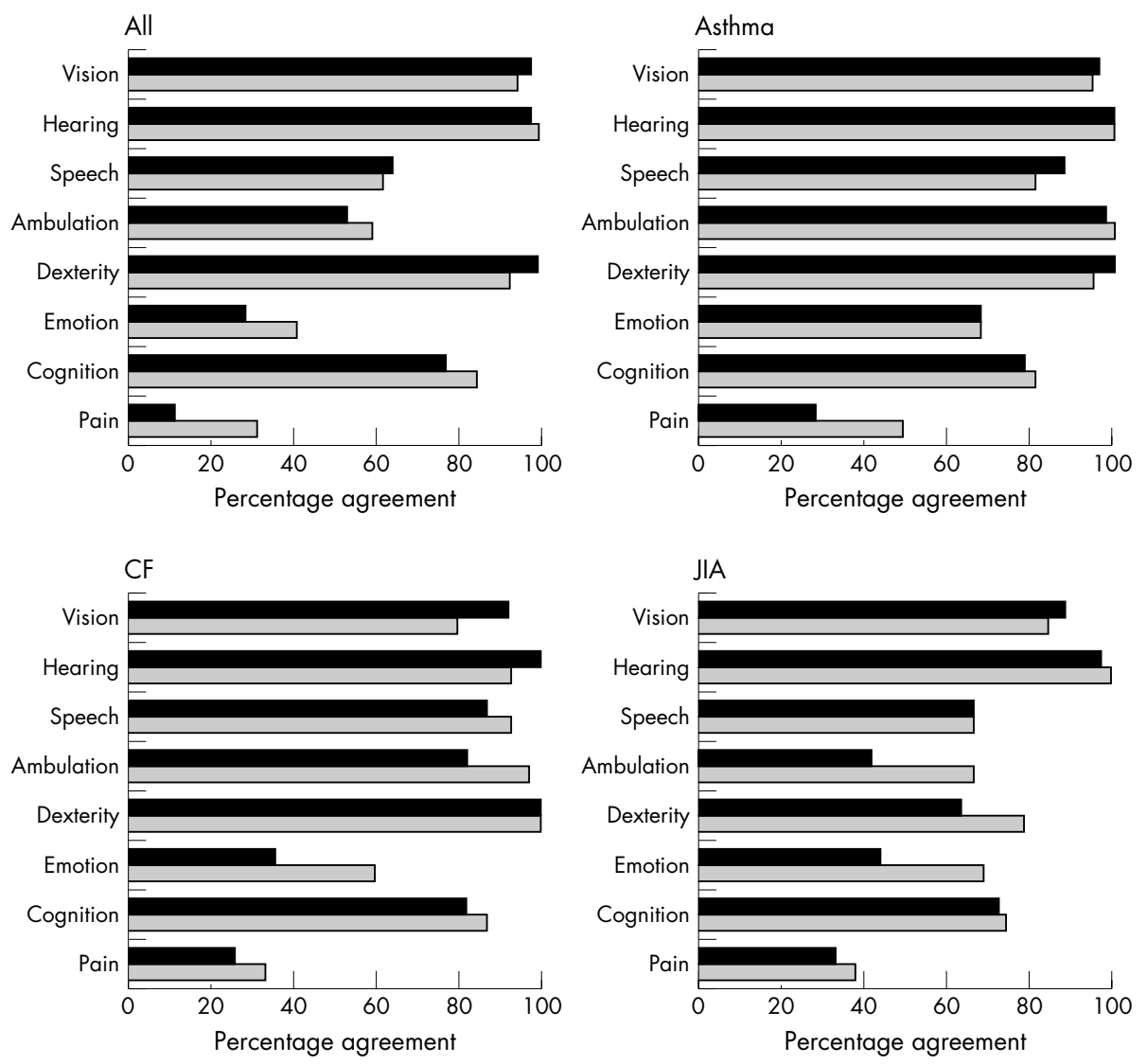

Agreement at baseline

Agreement at follow up

Figure 1 Percentage agreement between paediatricians and parents of chronically ill children (ALL, asthma, CF, and JIA) for each HUI3 attribute at baseline and follow up.

Positive values indicate the paediatrician assessing more impairment than the parents did, and negative values represent the parent assessing more impairment than the paediatrician did. The differences for the attributes vision, hearing, dexterity, and cognition were relatively small and are therefore not shown. For the attribute pain/discomfort the differences were most perspicuous. In all patient groups, at baseline and follow up, the parents assessed the patient to have more pain/discomfort than the paediatricians thought them to have.

\section{DISCUSSION}

We have found that differences in the perception of health between parents of children with a chronic disease and their paediatricians exist at diagnosis and after a period of follow up.

In general, the percentage agreement between parents and physicians for each patient group was found to be higher in the objective than in the subjective health attributes. Our earlier findings from the literature showed a pooled percentage agreement of $84-89 \%$ for the objective attributes and of $69-77 \%$ for the subjective domains. ${ }^{8}$ In the present study the agreement for the attribute pain/discomfort was remarkably low for all patient groups. For the attribute emotion, the agreement was lower than expected from the literature. ${ }^{8}$ Probably, in our study the agreement for these attributes was low because most patients consulted the physician in the acute phase of the disease, whereas in the literature the HUI has been used mostly in survivors of childhood cancer. ${ }^{15-20} 23-25$ It is known from the literature that QoL of patients can change dramatically during treatment. For example, the QoL of children with recurrent acute otitis media and/or chronic otitis media with effusion significantly improved after surgical intervention, ${ }^{30}$ whereas the QoL of children with chronic viral hepatitis temporarily deteriorated during alpha-interferon therapy. ${ }^{31}$ Also, differences in perception of QoL between parents and physicians and the magnitude of these differences are likely to be susceptible to change in time. In a study of adults with cancer it was found that the agreement for the more private domains (such as feelings, social functioning, overall QoL, and pain/discomfort) was slightly higher at follow up than at baseline. Firstly, it is suggested that monitoring the patients' QoL over time may increase the caregivers' awareness of wellbeing of the patient. ${ }^{32}$ Secondly, the objective domains are visible and more concrete than the subjective domains. Therefore, a higher agreement can be expected in the former. ${ }^{33}$ However, in our study the agreement did not essentially change over follow up periods, although patients were treated for their acute symptoms and patients and physicians became acquainted to each other. At follow up the rates of (dis)agreement remained fairly stable. Only for patients with asthma (pain/discomfort) and for patients with JIA (ambulation and emotion) did the agreement improve at follow up. We found obvious differences in four of the eight attributes. Paediatricians rated more emotional problems (ALL, CF, JIA) and problems with ambulation (JIA). Parents rated more pain/discomfort (all patient groups) and speech impairments. 

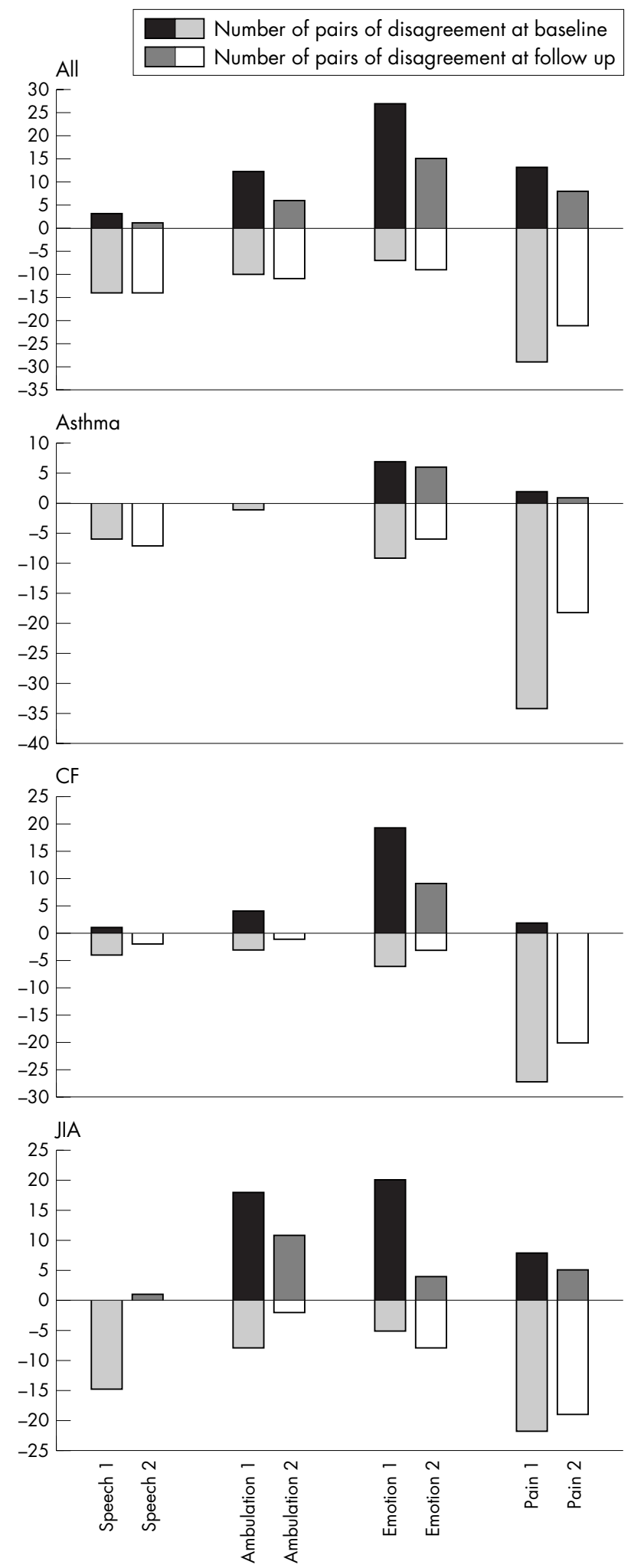

Figure 2 Number of pairs of disagreement between paediatricians and parents of chronically ill children (ALL, asthma, CF, and JIA) for the HUI3 attributes speech, ambulation, emotion, and pain/discomfort. The HUI 3 attribute levels of the parents were subtracted from the levels of the paediatricians. The positive bars represent the number of pairs for each attribute where the paediatrician scored a higher level of impairment within attributes than the parent at baseline $(1$; black) and at follow up (2; dark grey). The negative bars represent the number of pairs where the parent scored a higher level of disturbances within attributes than the paediatrician at baseline $(1 ;$ grey) and at follow up (2; white).
Table 2 Proportion and change of differences at baseline and follow up

\begin{tabular}{llll}
\hline Diagnosis & Attribute & $\boldsymbol{P}_{\mathbf{1}}-\boldsymbol{P}_{\mathbf{2}}$ & $\mathbf{9 5 \%} \mathrm{Cl} \boldsymbol{P}_{\mathbf{1}}-\boldsymbol{P}_{\mathbf{2}}$ \\
\hline Asthma & Pain/discomfort & 0.23 & 0.05 to 0.39 \\
JIA & Ambulation & 0.26 & 0.07 to 0.44 \\
JIA & Emotion & 0.28 & 0.08 to 0.48
\end{tabular}

The proportions of differences between parents and physicians at baseline $\left(P_{7}\right)$ and at follow up $\left(P_{2}\right)$ for each attribute were calculated. The differences in proportions at baseline and follow up were given by $P_{1}-P_{2}$, $95 \% \mathrm{Cl}$ were calculated using the formula $P_{1}-P_{2} \pm 1.96 * S E\left[P_{1}-P_{2}\right]^{29}$

Only attributes with $95 \% \mathrm{Cl}$ excluding zero were shown.

In our study we did not use the children's' assessment of their quality of life. However, for the attribute pain/ discomfort it is known from the literature that physicians have the tendency to underestimate the intensity of the patient's pain/discomfort. ${ }^{32}{ }^{34-36}$ A possible explanation for the disagreement on speech impairment is that in young children paediatricians tend to interpret HUI level 1 as language development according to age, while parents scored level 3 as development according to age.

Before further interpretation of our results, some methodological issues need to be addressed. A strong feature of our study is that the participation rate was high and that it had very few missing data at baseline. Furthermore, we used a validated instrument to measure quality of life as judged by both physicians and the parents. Physicians completed the assessments independent from parental HUI measurements. Some other points need attention. Complete follow up data were available for $80 \%$ of the patients. Because the differences in perception of QoL at baseline were the same for patients with and without complete follow up data, we expect our results to be valid. Each paediatrician $(n=37)$ completed the HUI several times (range 1-34). In 90 patients the same paediatricians completed the assessment both at baseline and at follow up. Due to a learning effect of the paediatrician the percentage agreement in this study might be higher than would have been found in regular clinical practice. The HUI has been validated originally for children of 6 years and older. ${ }^{13}$ For young children only a few QoL questionnaires are available (Functional Status, RAND, and the TAPQOL). ${ }^{37-39}$ In a study assessing the health status in a heterogeneous population of children admitted to intensive care, it seemed feasible to classify children over 1 year of age reliably, using the HUI by an observer well known to the patient. ${ }^{40}$ In our study the HUI3 questionnaire was administered to the parents by an interviewer at baseline and was self-completed at follow up while the HUI3 was selfcompleted by the paediatricians (both assessments). In the literature different results are reported about the effect of the mode of administration of HRQoL questionnaires. Grootendorst et al found that subjects participating in the Ontario Health Study, completing the interviewer administered form, reported less dysfunction than subjects completing the self-reported form. ${ }^{41}$ However Verrips et al reported that the mean number of affected attributes was higher and the mean utility score was lower when children (preterm born infants now 14 years old) and proxies were interviewed than when they self-completed the questionnaire. ${ }^{27}$ Both studies used the HUI3 to assess the health status. In our study the participating paediatricians preferred the selfcompleted form because of their time schedule at the outpatient clinic.

With regard to the implications of our study, we conclude that QoL should be given a fair amount of attention in chronic paediatric illnesses. Good medical practice requires an optimal relationship between physicians, patients, and 


\section{What is already known on this topic}

- Little is known in the literature about the differences in perception of quality of life between patients and physicians

- Objective attributes of health had a higher agreement between parents and physicians than the more subjective attributes

- The magnitude of the difference in perception of quality of life between parents and physicians is unknown

parents for mutual understanding of the impact of the disease. Whatever the reasons for discrepancies of perception, physicians should be aware that parents and physicians may look differently at the impact of the disease. This is particularly important for discrepancies pertaining to the attributes emotion and pain, which are vital to the child's wellbeing. Physicians have the tools to improve on this, by better and more explicitly discussing these issues with patients and parents. As differences in perception of QoL between parents and physicians may be different for various countries/populations, replication studies are needed. QoL evaluation may have a prominent role in assessing the changes of a patient's condition in the natural course of the disease or in the follow up of therapeutic interventions. In this way, better mutual understanding of perception of QoL between physician and the parents of the patient may enhance effects of treatment.

\section{ACKNOWLEDGEMENTS}

We are grateful to all parents of the patients and all clinicians of the participating centres for their efforts of taking part in this study. Special thanks are extended to E Visser and I van der Vaart, research nurses Sophia Children's Hospital, for their practical help, and to C Tims for her assistance with the data management.

\section{Authors' affiliations}

A J Janse, J L L Kimpen, Department of Paediatrics, Wilhelmina Children's Hospital, University Medical Centre Utrecht, Netherlands G Sinnema, Department of Paediatric Psychology, Wilhelmina Children's Hospital, University Medical Centre Utrecht, Netherlands C S P M Uiterwaal, Julius Centre for Health Sciences and Primary Care, University Medical Centre Utrecht, Netherlands

R J B J Gemke, Department of Paediatrics, VU University Medical Centre, Amsterdam, Netherlands

Role of the funding source: the sponsors of this study had no role in study design, data collection, data analysis, data interpretation, or writing of the report

Competing interests: none declared

\section{REFERENCES}

1 Rowbothan M, Harden N, Stacey B, et al. Gabapentin for the treatment of postherpetic neuralgia: a randomized controlled trial. JAMA 1998;280: 1837-42

2 Mant J, Carter J, Wade DT, et al. Family support for stroke: a randomised controlled trial. Lancet 2000;356:808-13.

3 Tetteroo E, Graaf van der Y, Bosch JL, et al. Randomised comparison of primary stent placement versus primary angioplasty followed by selective stent placement in patients with iliac-artery occlusive disease. Lancet 1998;351:1153-9.

4 Rougier $P$, Cutsum van $E$, Bajetts $E$, et al. Randomised trial of irinotecan versus fluorouracil by continuous infusion after fluorouracil failure in patients with metastatic colorectal cancer. Lancet 1998;352:1407-12.

5 Ghoname EA, Craig WF, Ahmed HE, et al. Percutaneous electrical nerve stimulation for low back pain: a randomized crossover study. JAMA 1999;281:818-23.

6 Bleyer WA. Cancer in older adolescents and young adults: epidemiology, diagnosis, treatment, survival, and importance of clinical trials. Med Pediatr Oncol 2002;38:1-10.

\section{What this study adds}

- Clear differences in perception of health and wellbeing between parents of children with a chronic disease and physicians were found

- In all patient groups, the physicians assessed the patient to have less pain/discomfort than the parents thought

- Quality of life may contribute to better understanding between physicians and parents

7 Cleeland CS, Gonin R, Hatfield AK, et al. Pain and its treatment in outpatients with metastatic cancer. N Engl J Med 1994;330:592-6.

8 Janse AJ, Gemke RJ, Uiterwaal CSPM, et al. Quality of life: patients' and doctors' don't always agree. A meta-analysis. J Clin Epidemiol 2004;57:653-61.

9 Feeny D, Leiper A, Barr RD, et al. The comprehensive assessment of health status in survivors of childhood cancer: application to high-risk acute lymphoblastic leukaemia. Br J Cancer 1993;67:1047-52.

10 Felder-Puig R, Frey E, Sonnleitner G, et al. German cross-cultural adaptation of the Health Utilities Index and its application to a sample of childhood cancer survivors. Eur J Pediatr 2000;159:283-8.

11 Barr RD, Furlong W, Dawson S, et al. An assessment of global health status in survivors of acute lymphoblastic leukemia in childhood. Am J Pediatr Hematol Oncol 1993;15:284-90.

12 stichting nederlandse werkgroep leukemie bij kinderen. Protocol SNWLKALL-9. Onderzoek en behandeling van kinderen met Acute Lymfatische Leukemie (ALL) 1999.

13 Feeny DH, Torrance GW, Furlong WJ. Health Utilities Index. In: SpilkerB, ed. Quality of life and pharmacoeconomics in clinical trials, 2nd edn. Philadelphia: Lippincott-Raven, 1996:239-52.

14 Feeny DH, Furlong WJ, Torrance GW, et al. Multiattribute and single-attribute utility functions for the health utilities index mark 3 system. Med Care 2002:40:113-28.

15 Barr RD, Pai MH, Weitzman S, et al. A multi-attribute approach to health status measurement and clinical management-illustrated by an application to brain tumors in childhood. Int J Oncol 1994;4:639-48.

16 Billson AL, Walker DA. Assessment of health status in survivors of cancer Arch Dis Child 1994;70:200-4.

17 Feeny D, Leiper A, Barr RD, et al. The comprehensive assessment of health status in survivors of childhood cancer: application to high-risk acute lymphoblastic leukaemia. Br J Cancer 1993;67:1047-52.

18 Felder-Puig R, Frey E, Sonnleitner G, et al. German cross-cultural adaptation of the Health Utilities Index and its application to a sample of childhood cancer survivors. Eur J Pediatr 2000;159:283-8.

19 Glaser AW, Davies K, Walker D, et al. Influence of proxy respondents and mode of administration on health status assessment following central nervous system tumours in childhood. Qual Life Res 1997;6:43-53.

20 Glaser AW, Abdul Rashid NF, U CL, et al. School behaviour and health status after central nervous system tumours in childhood. Br J Cancer 1997;76:643-50

21 Trudel JG, Rivard M, Dobkin PL, et al. Psychometric properties of the Health Utilities Index Mark 2 system in paediatric oncology patients. Qual Life Res 1998;7:421-32.

22 Barr RD, Petrie C, Furlong WJ, et al. Health-related quality of life during postinduction chemotherapy in children with acute lymphoblastic leukemia in remission: an influence of corticosteroid therapy. Int J Oncol 1997;11:333-9.

23 Barr RD, Simpson T, Whitton A, et al. Health-related quality of life in survivors of tumours of the central nervous system in childhood-a preference-based approach to measurement in a cross-sectional study. Eur $J$ Cancer 1999;35:248-55.

24 Glaser AW, Furlong W, Walker DA, et al. Applicability of the Health Utilities Index to a population of childhood survivors of central nervous system tumours in the U.K. Eur J Cancer 1999;35:256-61.

25 Le Gales C, Costet N, Gentet JC, et al. Cross-cultural adaptation of a health status classification system in children with cancer. First results of the French adaptation of the Health Utilities Index Marks 2 and 3. Int J Cancer Suppl 1999; 12:112-18.

26 Saigal S, Feeny D, Rosenbaum $P$, et al. Self-perceived health status and health-related quality of life of extremely low-birth-weight infants at adolescence. JAMA 1996;276:453-9.

27 Verrips GH, Stuifbergen MC, den Ouden AL, et al. Measuring health status using the Health Utilities Index: agreement between raters and between modalities of administration. J Clin Epidemiol 2001;54:475-81.

28 Gemke RJ, Bonsel GJ, van Vught AJ. Long-term survival and state of health after paediatric intensive care. Arch Dis Child 1995;73:196-201.

29 Altman DG. Practical statistics for medical research. In: Practical statistics for medical research. London: Chapman and Hall, 1996.

30 Richards M, Giannoni C. Quality-of-life outcomes after surgical intervention for otitis media. Arch Otolaryngol Head Neck Surg 2002;128:776-82.

31 lorio R, Pensati P, Botta S, et al. Side effects of alpha-interferon therapy and impact on health-related quality of life in children with chronic viral hepatitis. Pediatr Infect Dis J 1997;16:984-90. 
32 Sneeuw KC, Aaronson NK, Sprangers MA, et al. Value of caregiver ratings in evaluating the quality of life of patients with cancer. J Clin Oncol 1997; 15:1206-17.

33 Addington-Hall J, Kalra L. Measuring quality of life. Who should measure quality of life? BMJ 2001;322:1417-20.

34 Hodgkins M, Albert D, Daltroy L. Comparing patients' and their physicians' assessments of pain. Pain 1985;23:273-7.

35 Au E, Loprinzi CL, Dhodapkar M, et al. Regular use of a verbal pain scale improves the understanding of oncology inpatient pain intensity. J Clin Oncol 1994:12:2751-5.

36 Sneeuw KC, Aaronson NK, Sprangers MA, et al. Evaluating the quality of life of cancer patients: assessments by patients, significant others, physicians and nurses. Br J Cancer 1999:81:87-94.
37 Stein RE, Jessop DJ. Functional status II(R). A measure of child health status. Med Care 1990;28:1041-55.

38 Lewis CC, Pantell RH, Kieckhefer GM. Assessment of children's health status. Field test of new approaches. Med Care 1989;27:S54-65.

39 Fekkes M, Theunissen NC, Brugman E, et al. Development and psychometric evaluation of the TAPQOL: a health-related quality of life instrument for 1-5year-old children. Qual Life Res 2000;9:961-72.

40 Gemke RJ, Bonsel GJ. Reliability and validity of a comprehensive health status measure in a heterogeneous population of children admitted to intensive care. J Clin Epidemiol 1996;49:327-33.

41 Grootendorst PV, Feeny DH, Furlong W. Does it matter whom and how you ask? Inter- and intra-rater agreement in the Ontario Health Survey. J Clin Epidemiol 1997;50:127-35.

\section{Clinical Evidence-Call for contributors}

Clinical Evidence is a regularly updated evidence-based journal available worldwide both as a paper version and on the internet. Clinical Evidence needs to recruit a number of new contributors. Contributors are healthcare professionals or epidemiologists with experience in evidence-based medicine and the ability to write in a concise and structured way.

Areas for which we are currently seeking authors:

- Child health: nocturnal enuresis

- Eye disorders: bacterial conjunctivitis

- Male health: prostate cancer (metastatic)

- Women's health: pre-menstrual syndrome; pyelonephritis in non-pregnant women

However, we are always looking for others, so do not let this list discourage you.

Being a contributor involves:

- Selecting from a validated, screened search (performed by in-house Information Specialists) epidemiologically sound studies for inclusion.

- Documenting your decisions about which studies to include on an inclusion and exclusion form, which we keep on file.

- Writing the text to a highly structured template (about 1500-3000 words), using evidence from the final studies chosen, within 8-10 weeks of receiving the literature search.

- Working with Clinical Evidence editors to ensure that the final text meets epidemiological and style standards.

- Updating the text every six months using any new, sound evidence that becomes available. The Clinical Evidence in-house team will conduct the searches for contributors; your task is simply to filter out high quality studies and incorporate them in the existing text.

- To expand the topic to include a new question about once every 12-18 months.

If you would like to become a contributor for Clinical Evidence or require more information about what this involves please send your contact details and a copy of your CV, clearly stating the clinical area you are interested in, to Klara Brunnhuber (kbrunnhuber@ bmigroup.com).

\section{Call for peer reviewers}

Clinical Evidence also needs to recruit a number of new peer reviewers specifically with an interest in the clinical areas stated above, and also others related to general practice. Peer reviewers are healthcare professionals or epidemiologists with experience in evidence-based medicine. As a peer reviewer you would be asked for your views on the clinical relevance, validity, and accessibility of specific topics within the journal, and their usefulness to the intended audience (international generalists and healthcare professionals, possibly with limited statistical knowledge). Topics are usually 1500-3000 words in length and we would ask you to review between 2-5 topics per year. The peer review process takes place throughout the year, and our turnaround time for each review is ideally 10-14 days.

If you are interested in becoming a peer reviewer for Clinical Evidence, please complete the peer review questionnaire at www.clinicalevidence.com or contact Klara Brunnhuber (kbrunnhuber@bmigroup.com). 